\title{
Flora vascular del Parque Futangue, Región de Los Ríos (Chile)
}

\section{Vascular flora of Futangue Park, Región de Los Ríos (Chile)}

\author{
Ricardo Moreno ${ }^{1 *}$, Carlos Le Quesne ${ }^{1}$, Iván Díaz ${ }^{1}$ \& Roberto Rodríguez ${ }^{2}$ \\ ${ }^{1}$ Instituto de Silvicultura, Universidad Austral de Chile, Casilla 567, Isla Teja s/n, Valdivia, Chile. \\ 2Departamento de Botánica, Facultad de Ciencias Naturales y Oceanográficas, Universidad de Concepción, Casilla 160-C, \\ Concepción. \\ *richo.m@gmail.com
}

\begin{abstract}
RESUMEN
Se presentan los resultados del primer estudio florístico del Parque Futangue, un Área Silvestre Protegida (ASP) de los Bosques Templados del Sur de Sudamérica (BTSS), ubicado en la Cordillera de los Andes (40² 25' S; 72 $16^{\prime}$ O), próximo al complejo volcánico Puyehue-Cordón del Caulle, Región de Los Ríos. Se identificaron 295 especies, pertenecientes a 91 familias y 165 géneros. Este parque representa una muestra importante de los BTSS (ca. 40-70\%). La flora de Futangue se caracteriza por un $66 \%$ de las especies de origen nativo, $8 \%$ catalogadas con problemas de conservación, con un $19 \%$ de especies endémicas de Chile. La riqueza de especies superó lo esperado con respecto a otras áreas silvestres cercanas. La escasa proporción de especies introducidas y la baja cobertura observada de éstas indicarían que Futangue presenta una baja alteración antrópica. Este reporte contribuye a llenar un vacío de registros para la zona y da cuenta de la importancia de mantener actualizado los listados de flora para planes de manejo y conservación en ASP.
\end{abstract}

Palabras Clave: Diversidad, representatividad, endemismo, estado de conservación.

\begin{abstract}
We presented the checklist of the vascular flora of the Futangue Park, a private natural protected area, in the Andean Temperate Region of southern Chile. We recorded 295 species, distributed in 91 families and 165 genera. This park support an important proportion of the flora from temperate forests of southern South America (ca. 40-70\%). The 66\% of plant species living in the park were native, with an $8 \%$ of the flora considered as threatened at national level. A high percentage of endemic species (19\%) characterized the flora of the Futangue Park. The species richness was higher than expected based on the knowledge from nearby areas. Also, few and scarce exotic species were found, which also indicates this area is well preserved. Our report contributes to fill the gaps in the knowledge of plant richness and its distribution in the Andes, providing important information for the conservation and management of this and other protected areas.
\end{abstract}

KeYword: Diversity, representativeness, endemism, conservation status.

\section{INTRODUCCIÓN}

El conocimiento de la composición florística de las áreas protegidas provee antecedentes para entender la estructura, dinámica y diversidad actual, siendo fundamental para determinar el valor de conservación de éstas (Lindenmayer \& Franklin 2002, Primack et al. 2001). Los inventarios florísticos contribuyen a la planificación y desarrollo de estrategias de conservación y uso sustentable de los ecosistemas (Squeo et al. 2008, Oltremari 2003, Ter Steege et al. 2000, Van Andel 2001, Becerra \& Cruz 2000). La información que se tiene de la diversidad vegetal en Chile comprende documentos de una amplia escala geográfica
(Gajardo 1994, Pisano 1956, Schmithüsen 1956, Oberdorfer 1960), basados en numerosos trabajos a escala local. Sin embargo, el grado de conocimiento de la flora varía entre áreas biogeográficas (Squeo et al. 1998, Becerra \& Cruz 2000), con un consecuente sesgo del grado de información. A escala de regiones geopolíticas se encuentran los trabajos de Squeo et al. (2008, 2001) (Región de Atacama y Región de Coquimbo, respectivamente) y Serey et al. (2007) (Región de O'Higgins), los que han logrado detectar la riqueza de flora y estado de conservación en estas regiones. Se considera que en Chile la información de base que describe la diversidad de flora en las Áreas Silvestres Protegidas (ASP) es aún escasa (Becerra \& Cruz 2000), 
incompleta y sesgada (Simonetti et al. 1995), o que existe un conocimiento deficiente sobre la representatividad de la flora y vegetación en áreas protegidas (Luebert \& Becerra 1998).

En la Región de los Ríos, las ASP privadas abarcan alrededor de 170 mil hectáreas $(9,2 \%$ de la superficie regional), mientras que la superficie del Sistema Nacional de Áreas Protegidas del Estado (SNASPE) alcanza sólo a 75.509 hectáreas ( $c a .4 \%$ ). Dentro de las ASP privadas se encuentra el Parque Futangue, está ubicado en la Cordillera de los Andes ( $\left.40^{\circ} 25^{\prime} \mathrm{S} ; 7^{\circ} 16^{\prime} \mathrm{O}\right)$, próximo al complejo volcánico Puyehue-Cordón del Caulle. Es creado en 1997 y forma parte de una red de ASP privadas. El objetivo de este parque es conservar la flora, la fauna y los servicios ecosistémicos del área. Está cubierto principalmente por bosque nativo adulto, característico de los Bosques Templados del Sur de Sudamérica (BTSS), incluyendo cinco tipos forestales (Donoso 1981) (Figura 1). A pesar de su extensión y cobertura de bosque nativo, se desconoce la riqueza de especies presentes en el área, y cuanto de la flora de la región está siendo conservada en Futangue. En consecuencia, el objetivo de este artículo es documentar la riqueza florística del parque, sus formas de crecimiento, origen geográfico, con el fin de analizar su valor de conservación en el contexto regional y nacional.
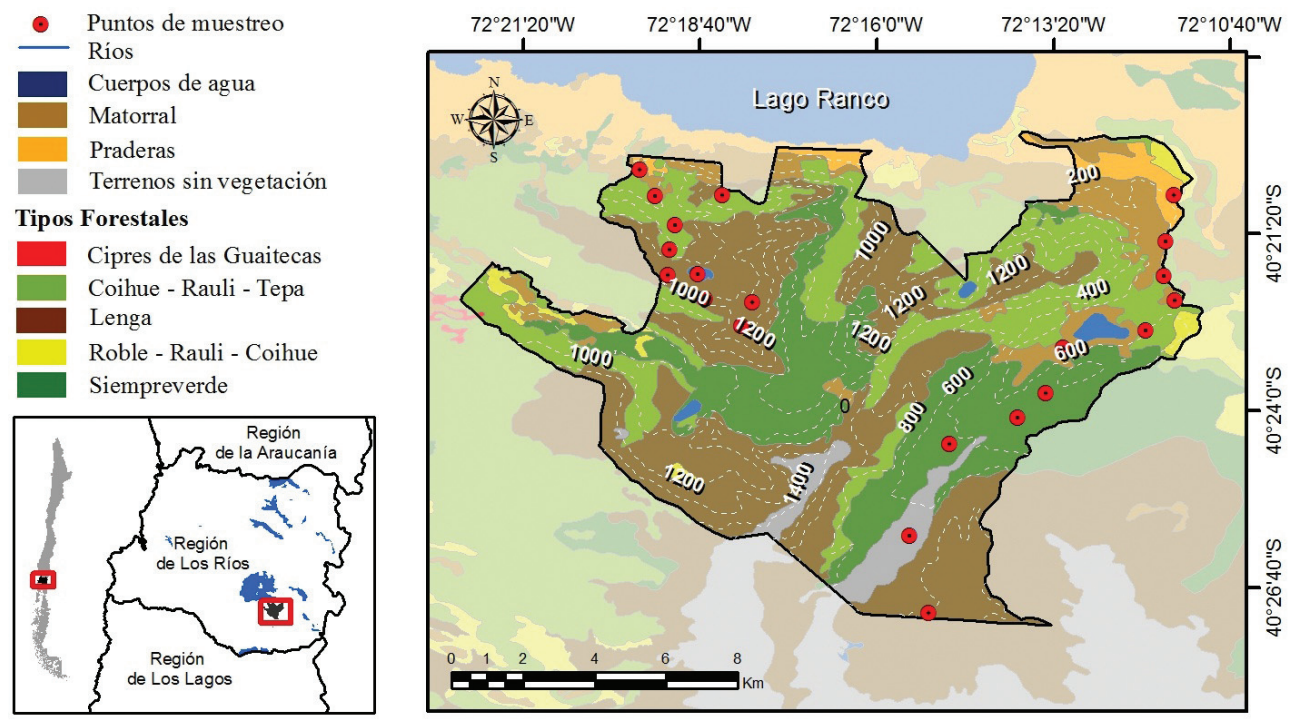

Figura 1. Ubicación Parque Futangue. Puntos de muestreo indicado con puntos rojos distribuidos a lo largo del gradiente altitudinal.

FIGURE 1. Location of the Futangue Park. Sample point is indicated with red points distributed along the altitudinal gradient.

\section{MATERIALES Y MÉTODOS}

\section{Área DE ESTUdio}

El Parque Futangue está ubicado en la precordillera andina de la comuna de Lago Ranco (aproximadamente $40^{\circ} 20^{\circ}$ $40^{\circ} 30^{`} \mathrm{~S} ; 72^{\circ} 13^{`}-72^{\circ} 21^{`} \mathrm{O}$ ), en la Región de los Ríos. Al norte limita con el lago Ranco, al sur colinda con el Parque Nacional Puyehue en la zona del Volcán Puyehue y el Cordón montañoso del Caulle. Al este continúa la Cordillera de los Andes y al oeste con serranías bajas de la precordillera cercana a la depresión central (Fig. 1). Futangue posee una superficie de 12.500 ha aproximadamente y abarca un gradiente altitudinal desde los $200 \mathrm{~m}$ s.n.m hasta $1.350 \mathrm{~m}$ s.n.m. El tipo de clima corresponde a un macrobioclima templado, mesotemplado húmedo a supratemplado hiperhúmedo, oceánico (Luebert \& Pliscoff 2006). La precipitación media anual fluctúa de 2.500 a $4.000 \mathrm{~mm}$. En las zonas más altas la precipitación se deposita en forma de nieve cubriendo durante 4 a 6 meses el área. La temperatura media anual es de 6 a $9{ }^{\circ} \mathrm{C}$, sin embargo, sobre la cota 1.100, se estiman temperaturas mínimas bajo $0{ }^{\circ} \mathrm{C}$ gran parte del año (Muñoz 1980).

\section{Metodología}

El muestreo se basó en prospecciones botánicas donde se realizó un recorrido aleatorio en una superficie aproximada de $0,5 \mathrm{~km}^{2}$, abarcando distintos ambientes a lo largo de un día. Los puntos de muestreo fueron dirigidos según unidades de vegetación, siguiendo el gradiente altitudinal (Fig. 1). Se 
realizaron dos transectos: en el primero se establecieron 10 puntos de muestreo entre $200-1.000$ m s.n.m. y en el segundo 11 puntos de muestreo entre $400-1.350 \mathrm{~m}$ s.n.m. El distanciamiento entre puntos en ambos casos fue irregular. Los muestreos se efectuaron durante el verano de 2008, 2009 y 2010 , acumulando alrededor de 60 días de trabajo de terreno, junto a una campaña de una semana en la primavera de 2010. Las muestras recolectadas fueron herborizadas y depositadas en la colección de muestras de flora de la Facultad de Ciencias Forestales y Recursos Naturales de la Universidad Austral de Chile. Las especies fueron determinadas con antecedentes bibliográficos y luego comparadas con muestras del Herbario de la Universidad de Concepción (CONC). La nomenclatura y la forma de crecimiento siguen a Zuloaga et al. (2009). Sin embargo, la forma de crecimiento se simplificó usando las categorías: árbol, arbusto, hierba, epífita, parásitas, trepadoras y acuática. El origen geográfico se clasificó en tres categorías, a partir de la distribución geográfica indicada por Zuloaga et al. (2009): introducida, nativa de los BTSS y endémicas de la vertiente pacífica de la Cordillera de los Andes. El estado de conservación de toda la flora considera a Benoit et al. (1989), la versión 2.3 de UICN (Hechenleitner et al. 2005), y para Pteridophyta de acuerdo a Baeza et al. (1998) y Rodríguez et al. (2009).

\section{RESULTADOS}

\section{RiQueZA TAXONÓMICA}

La flora vascular del Parque Futangue asciende a 295 especies, distribuidas en 91 familias y 165 géneros (Tabla I). Destaca la participación de Magnoliopsida con un 65,1\% como el grupo mejor representado. El segundo grupo más importante corresponde a Liliopsida $(17,6 \%)$, seguido por Pteridophyta $(15,9 \%)$ y finalmente Pinophyta con sólo 4 especies $(1,4 \%)$. Esta proporción es similar a nivel de géneros, sin embargo a nivel de familias la importancia entre Pteridophyta y Liliopsida se invierte, es decir la riqueza de familias de Pteridophyta es mayor (Tabla I).

Entre las Magnoliopsida la familia más numerosa es Asteraceae, con 45 especies y 22 géneros. Luego siguen las Myrtaceae, con 10 especies pertenecientes a 6 géneros. Scrophulariaceae con 11 especies y 5 géneros. Se destacan también las familias Nothofagaceae y Berberidaceae, ambas con 6 especies y un género (Nothofagus y Berberis, respectivamente) (Anexo). La clase Liliopsida esta representada principalmente por la familia Poaceae con 25 especies distribuidas en 15 géneros. La mayoría de las especies de este género son herbáceas, a excepción de las pertenecientes al género Chusquea. Otra familia importante de las Liliopsida es Orchidaceae, con 5 especies y 3 géneros (Anexo). En la división Pteridophyta la familia más numerosa es Hymenophyllaceae con 15 especies y 3 géneros, seguida por la familia Blechnaceae con 10 especies, distribuidas en un solo género: Blechnum. Finalmente, las Pinophyta están representadas por dos familias: Podocarpaceae, con tres especies Podocarpus salignus, P. nubigenus, Saxegothaea conspicua y Cupressaceae, con la especie Pilgerodendron uviferum (Anexo).

\section{Forma De CRECIMIENTO}

La forma de crecimiento dominante en Futangue es la herbácea con 162 especies (55\%), luego los arbustos con 49 especies $(16,6 \%)$, epífitas 27 especies $(9,2 \%)$, trepadoras 11 especies $(3,7 \%)$, parásitas 6 especies $(2 \%)$ y árboles 35 especies $(11,9 \%)$ y las acuáticas con sólo 5 especies $(1,7 \%)$ (Tabla II). La familia dominante para el hábito herbáceo es Asteraceae. En los arbustos están bien representadas las familias: Asteraceae (5 spp., género Baccharis), Ericaceae (4 spp., género Gaultheria), Berberidaceae (6 spp., género Berberis) y Escalloniaceae (4 spp., género Escallonia) (Tabla II). Entre la forma de crecimiento arbórea las familias más importantes son: Nothofagaceae y Myrtaceae. Entre las epífitas la familia con mayor riqueza es Hymenophyllaceae, con 14 especies en tres géneros (Hymenophyllum, Hymenoglossum y Serpyllopsis). Las plantas acuáticas están representadas por 5 especies de 4 familias (Tabla II).

\section{Origen Geográfico}

Respecto al origen geográfico de las especies, las proporciones se distribuyen como sigue: $66 \%$ nativas (196 spp), 19\% endémicas (56 spp) y 15\% introducidas (43 spp) (Tabla II). Las especies introducidas registradas en Futangue son principalmente herbáceas y equivalen al 10\% del total de las plantas introducidas en Chile continental (Matthei 1995; Castro et al. 2005). Tanto las especies endémicas como las nativas la mayoría son herbáceas (19 spp y 107 spp respectivamente) (Tabla II). Destaca la ausencia de especies epífitas que sean introducidas.

\section{DISCUSION}

RiQueZA DE ESPECIES

Con respecto a la flora de Chile, con $c a .5 .200$ especies (Marticorena 1991) Futangue representa un 5,7\%. Para los BTSS se reconoce una diversidad de flora vascular entre 400 (Smith-Ramírez et al. 2005) y 700 especies (Arroyo et al. 1996), por lo tanto la Flora del Parque Futangue representaría entre un $40-70 \%$ de la flora de los BTSS. Los relevamientos de flora vascular en las áreas andinas de los BTSS son relativamente escasos. El Parque Nacional Puyehue, colindante con el límite sur de Futangue, registra 260 especies en una superficie de 107.000 ha, abarcando un gradiente desde los $250 \mathrm{~m}$ s.n.m hasta $2.236 \mathrm{~m}$ s.n.m (Muñoz 1980). En el Parque Nacional Vicente Pérez 
Gayana Bot. 70(1), 2013

TABLA I. Número de familias, géneros y especies por grupo taxonómico de la flora vascular presente en el Parque Futangue

TABLE I. Number of families, genus and species for taxonomic group composing the vascular flora of Futangue Park.

\begin{tabular}{lcccc}
\hline Division & FAMILIAS & GÉNEROS & ESPECIES & $\%$ \\
\hline Pteridophyta & 15 & 20 & 47 & 15,9 \\
Pinophyta & 2 & 3 & 4 & 1,4 \\
Magnoliophyta & & & 192 & 65,1 \\
$\quad$ Magnoliopsida & 64 & 113 & 52 & 17,6 \\
$\quad$ Liliopsida & 10 & 29 & 295 & 100 \\
\hline Total & 91 & 165 & & \\
\hline
\end{tabular}

TABLA II. Riqueza de especies distribuidas por formas de crecimiento y divididas según origen geográfico.

TABLE II. Species richness by life form and by geographic origin.

\begin{tabular}{lcccc}
\hline & \multicolumn{3}{c}{ ORIGEN GEOGRÁFICO } & \multirow{2}{*}{ TotaL } \\
\cline { 2 - 4 } Forma CRECIMIENTO & $\mathrm{N}$ & $\mathrm{I}$ & $\mathrm{E}$ & $35(12 \%)$ \\
\hline árbol & 25 & 2 & 8 & $49(17 \%)$ \\
arbusto & 29 & 3 & 17 & $27(9 \%)$ \\
epífita & 19 & - & 8 & $12(4 \%)$ \\
trepadoras & 8 & - & 3 & $6(2 \%)$ \\
parásitas & 5 & - & 1 & $162(55 \%)$ \\
hierba & 107 & 36 & - & $5(2 \%)$ \\
acuática & 3 & 2 & $56(19 \%)$ & 295 \\
\hline Total & $196(66 \%)$ & $43(15 \%)$ & & \\
\hline
\end{tabular}

Rosales, de 253.789 ha, Martínez (1985) encontró 152 especies, en un gradiente de elevación entre los 150 y 3.470 m s.n.m. (Volcán Tronador). Por otra parte, en la Reserva Nacional Malalcahuello, Becerra \& Faúndez (1999) registraron 211 especies en una superficie de 12.789 ha. Dada las características de estos parques, vale decir, que están distribuidos en amplios gradientes altitudinales y en grandes superficies, poseen una similar historia biogeográfica y tipos forestales, y además, probablemente una alta similitud de flora, se esperaría encontrar una mayor riqueza en estos lugares. Lo anterior se plantea, ya que en Futangue al aumentar el esfuerzo de muestreo en alrededor de 25 puntos la curva se estabiliza en torno a 400 especies (Moreno 2011). Sin embargo se requieren nuevos estudios que permitan reconocer con precisión la riqueza y composición de estos bosques.

\section{ORIGEN GEOGRÁFICO}

El origen fitogeográfico de las especies es un elemento que permite evaluar el grado de alteración antrópica (Hauenstein et al.1988). En las ASP: P.N. Puyehue (Muñoz 1980), P.N. Cerro Nielol y P.N. Tolhuaca (Hauenstein et al.1988), R.N.
Malalcahuello (Becerra \& Faúndez 1999), P.N. Pali Aike (Domínguez et al. 2004), R.N. Alto Bío-Bío (FuentesRamírez et al. 2011), se observa que la proporción de especies introducidas puede variar entre 11 y 40\% (18 y 95 especies). De acuerdo a la escala de evaluación propuesta por González (2000), y considerando que las especies introducidas dentro del parque se concentran en zonas bajas (< 400m s.n.m.) a lo largo de caminos y senderos $(<1 \%$ de la superficie del Parque), se puede catalogar a Futangue como poco intervenido.

En Futangue se catastraron 56 especies endémicas de la vertiente occidental de los Andes (Tabla II), representando cerca de 3\% de la flora endémica de Chile. En contraste, en el P.N. Puyehue se registran 33 especies endémicas exclusivas de la vertiente occidental de los Andes y 138 de los BTSS (Muñoz 1980). En la Cordillera de la Costa se han registrado hasta 44 especies arbóreas endémicas de los BTSS (Smith-Ramírez et al. 2005) mientras que en Futangue son 33 especies, de las cuales 8 son endémicas de la vertiente occidental de los Andes (Tabla II). Esto, sumado a la diferencia en su historia biogeográfica, sugiere que 
para otras formas de crecimiento la riqueza y el número de especies endémicas deberían ser mayores en la Cordillera de la Costa (Smith-Ramírez et al. 2005).

\section{Estado de Conservación}

De las especies registradas en Futangue, 29 han sido clasificadas en alguna categoría de conservación (Tabla III), lo que equivale a decir que cerca del $90 \%$ de la flora no ha sido clasificada o no calificaría en algún grado de amenaza. De las especies categorizadas 8 son leñosas arborescentes, las restantes son Pteridophyta herbáceas (Tabla III). El grado de amenaza está en parte relacionado con el conocimiento general que se tiene de una especie, conocimiento bastante escaso para herbáceas y epífitas. Las especies Eucryphia cordifolia Cav., Nothofagus dombeyi (Mirb.) Oerst. y Laureliopsis philippiana (Looser) Schodde son categorizadas por la UICN como de "Riesgo Bajo", y Pilgerodendron uviferum (D. Don) Florin considerada "Vulnerable", de acuerdo al criterio de disminución de la superficie de hábitat. Paradojalmente, en la Región de los Ríos estas especies se concentran en áreas de baja altitud que se encuentran escasamente resguardadas por el SNASPE. Si estas especies emblemáticas, que se consideran abundantes en zonas bajas, fuesen integradas en un sistema eficiente para la conservación tendrían un efecto de "paraguas" (Possingham et al. 2006) sobre muchas otras especies menos conspicuas y desconocidas.

TABLA III. Especies catalogadas con problemas de conservación.

TABLE III. Red list of threatened species.

\begin{tabular}{|c|c|c|}
\hline ESPECIE & FAMILIA & CATEGoría de Conservación \\
\hline Blechnum corralense & Blechnaceae & En Peligro ${ }^{3,4}$ \\
\hline Fascicularia bicolor & Bromeliaceae & Vulnerable $^{1}$ \\
\hline Asplenium trilobun & Aspleniaceae & Vulnerable ${ }^{3,4}$ \\
\hline Blechnum asperum & Blechnaceae & Vulnerable ${ }^{3,4}$ \\
\hline Blechnum blechnoides & Blechnaceae & Vulnerable 3,4 \\
\hline Elaphoglossum gayanum & Lomariopsidaceae & Vulnerable ${ }^{4}$ Rara $^{3}$ \\
\hline Grammitis magellanica & Grammitidaceae & Vulnerable 3,4 \\
\hline Hymenoglossum cruentum & Hymenophyllaceae & Vulnerable ${ }^{3,4}$ \\
\hline Hymenophyllum caudiculatum & Hymenophyllaceae & Vulnerable ${ }^{3,4}$ \\
\hline Hymenophyllum cuneatum & Hymenophyllaceae & Vulnerable ${ }^{3,4}$ \\
\hline Hymenophyllum dicranotrichum & Hymenophyllaceae & Vulnerable ${ }^{3,4}$ \\
\hline Hymenophyllum tortuosum & Hymenophyllaceae & Vulnerable ${ }^{3,4}$ \\
\hline Isoëtes chubutiana & Isoetaceae & Vulnerable ${ }^{3,4}$ \\
\hline Lophosoria quadripinnata & Dicksoniaceae & Vulnerable ${ }^{3,4}$ \\
\hline Lycopodium confertum & Lycopodeaceae & Vulnerable ${ }^{4}$ Rara $^{3}$ \\
\hline Lycopodium paniculatum & Lycopodeaceae & Vulnerable $^{3,4}$ \\
\hline Pilgerodendron uviferum & Cupressaceae & Vulnerable $^{2}$ \\
\hline Podocarpus salignus & Podocarpaceae & Vulnerable $^{2}$ \\
\hline Pleopeltis macrocarpa & Polypodiaceae & Vulnerable $^{3,4}$ \\
\hline Saxegothaea conspicua & Podocarpaceae & Casi Amenazada ${ }^{2}$ \\
\hline Podocarpus nubigena & Podocarpaceae & Casi Amenazada ${ }^{2}$ \\
\hline Aextoxicon punctatum & Aextoxicaceae & Datos Insuficientes $^{2}$ \\
\hline Blechnum arcuatum & Blechnaceae & Datos Insuficientes ${ }^{3,4}$ \\
\hline Greigia landbeckii & Bromeliaceae & Datos Insuficientes ${ }^{1}$ \\
\hline Hypolepis poeppigii & Dennstaedtiaceae & Datos Insuficientes ${ }^{3,4}$ \\
\hline Serpillopsis caespitosa & Hymenophyllaceae & Datos Insuficientes ${ }^{3,4}$ \\
\hline Eucryphia cordifolia & Eucryphiaceae & Preocupación menor ${ }^{2}$ \\
\hline Laurelia sempervirens & Monimiaceae & Preocupación menor ${ }^{2}$ \\
\hline Nothofagus dombeyi & Nothofagaceae & Preocupación menor ${ }^{2}$ \\
\hline
\end{tabular}

${ }^{1}$ Benoit 1989; ${ }^{2}$ versión 2010.4 de UICN (Hechenleitner et al. 2005); ${ }^{3}$ Baeza et al. 1998, ${ }^{4}$ Rodríguez et al. 2009. 


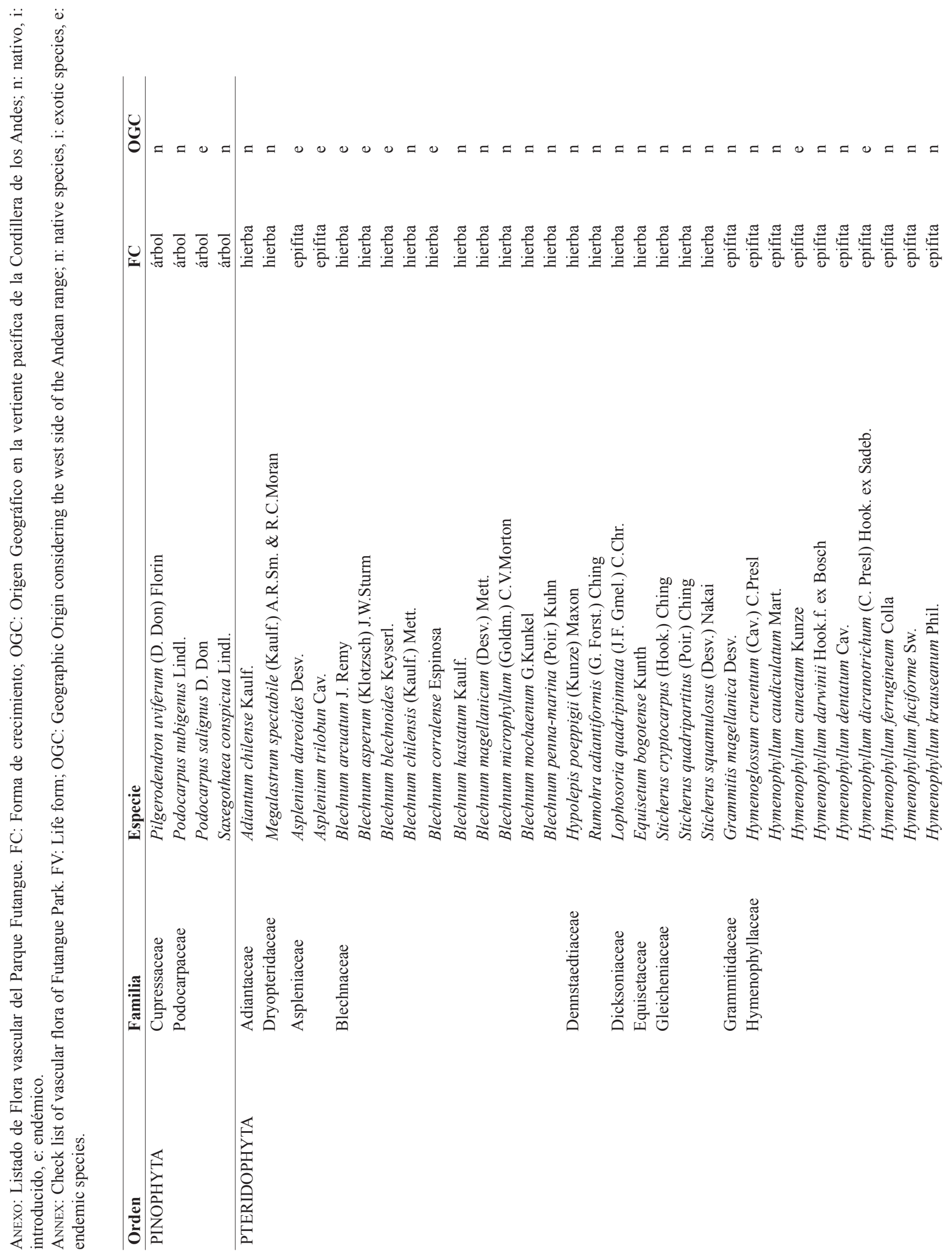




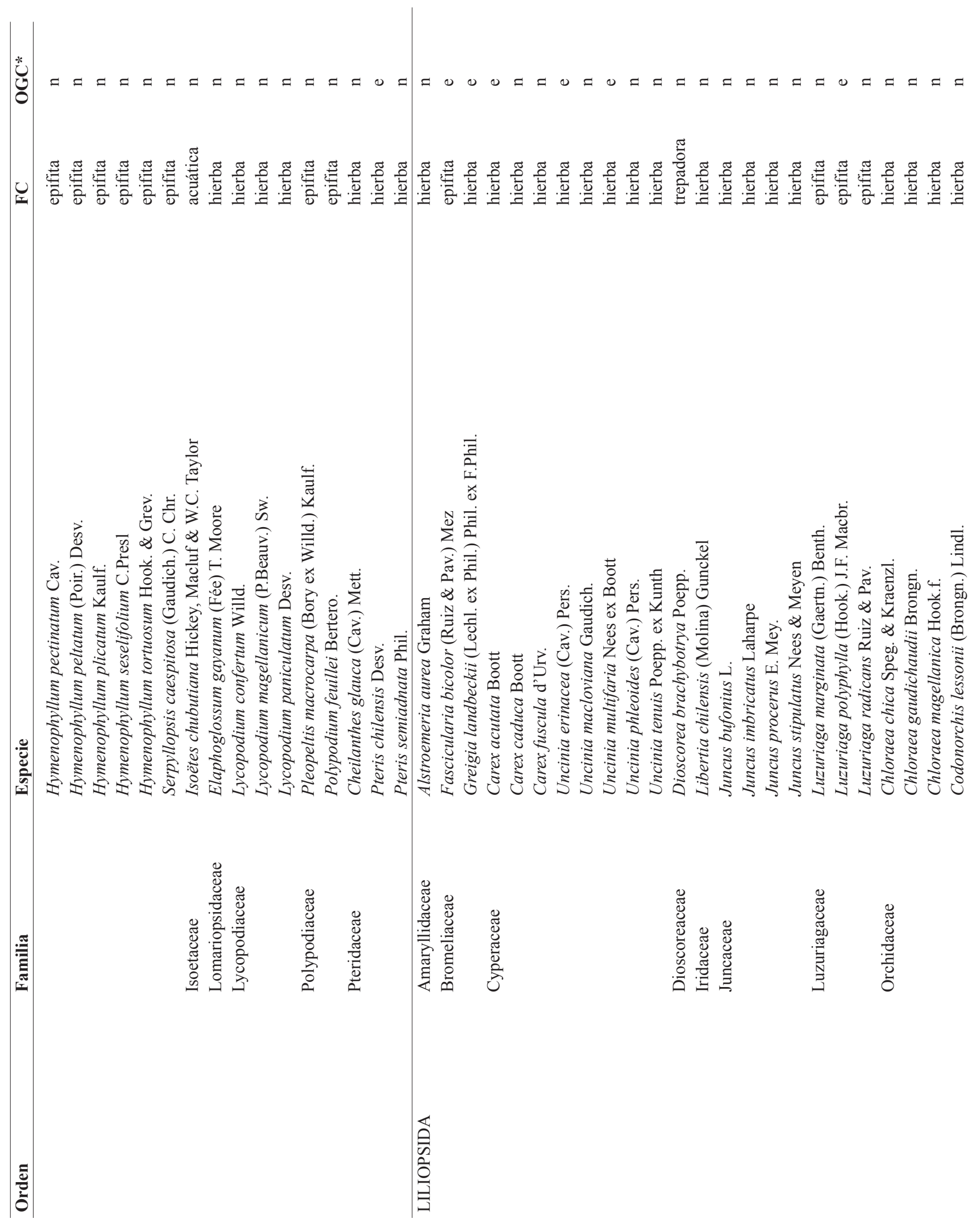




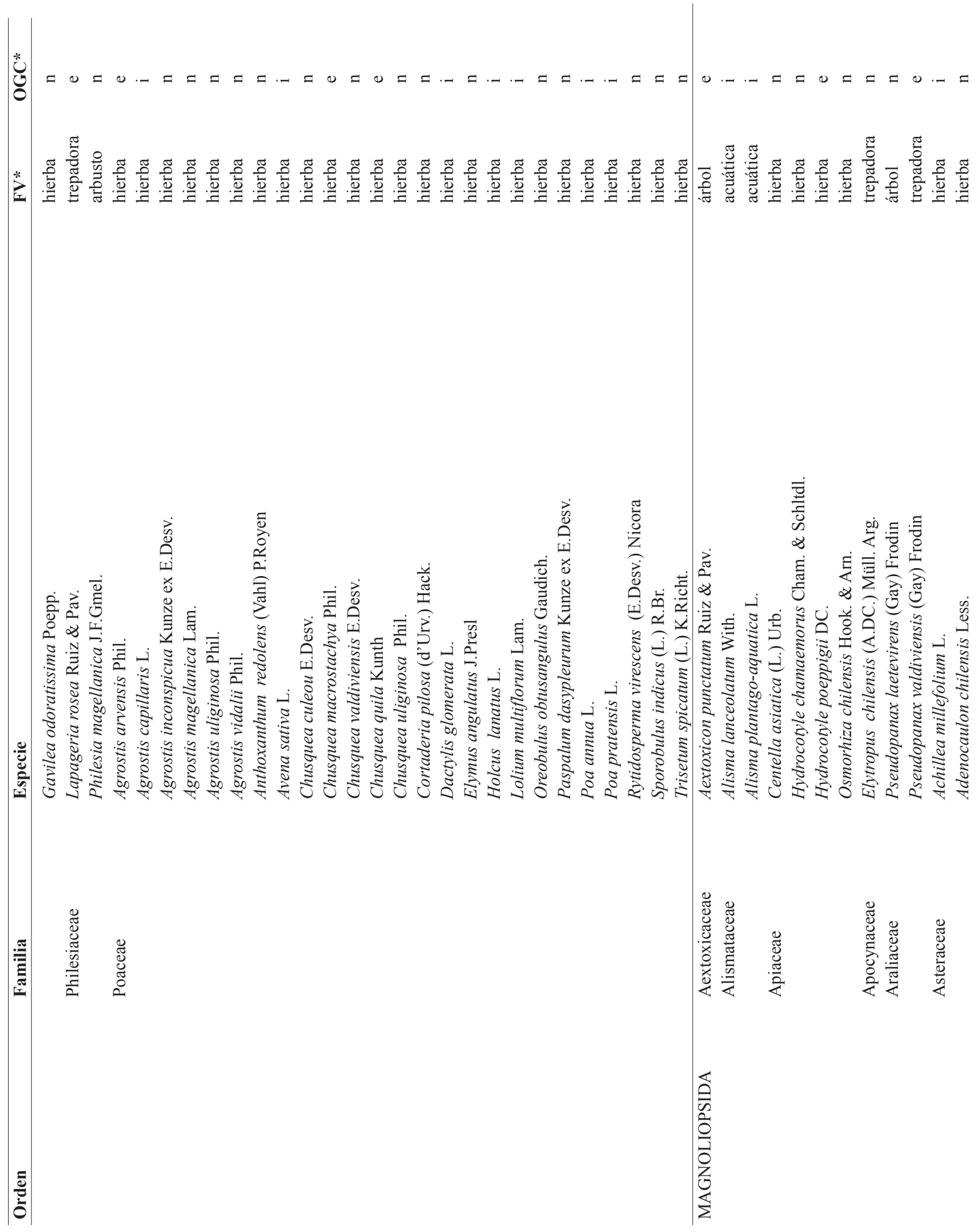




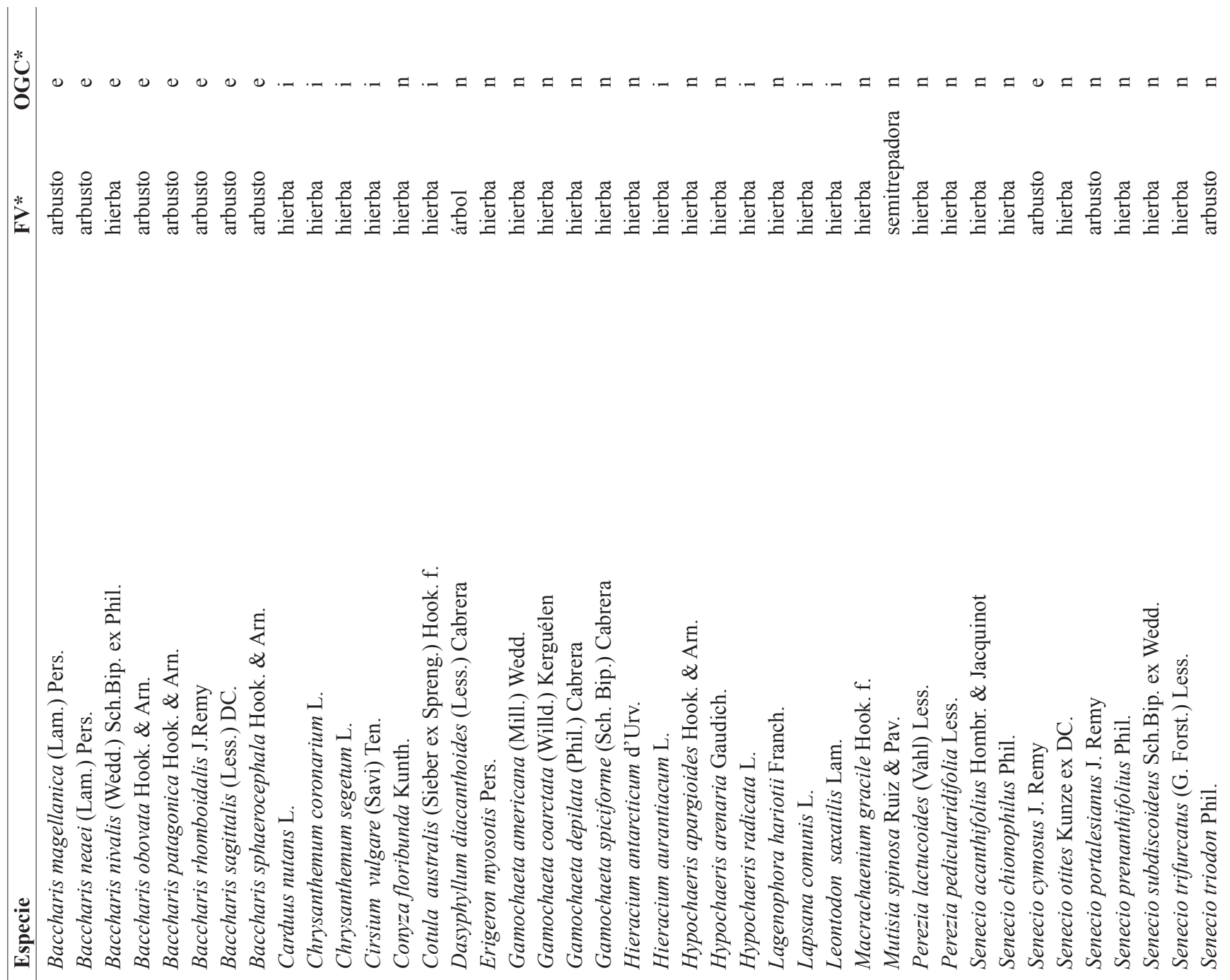




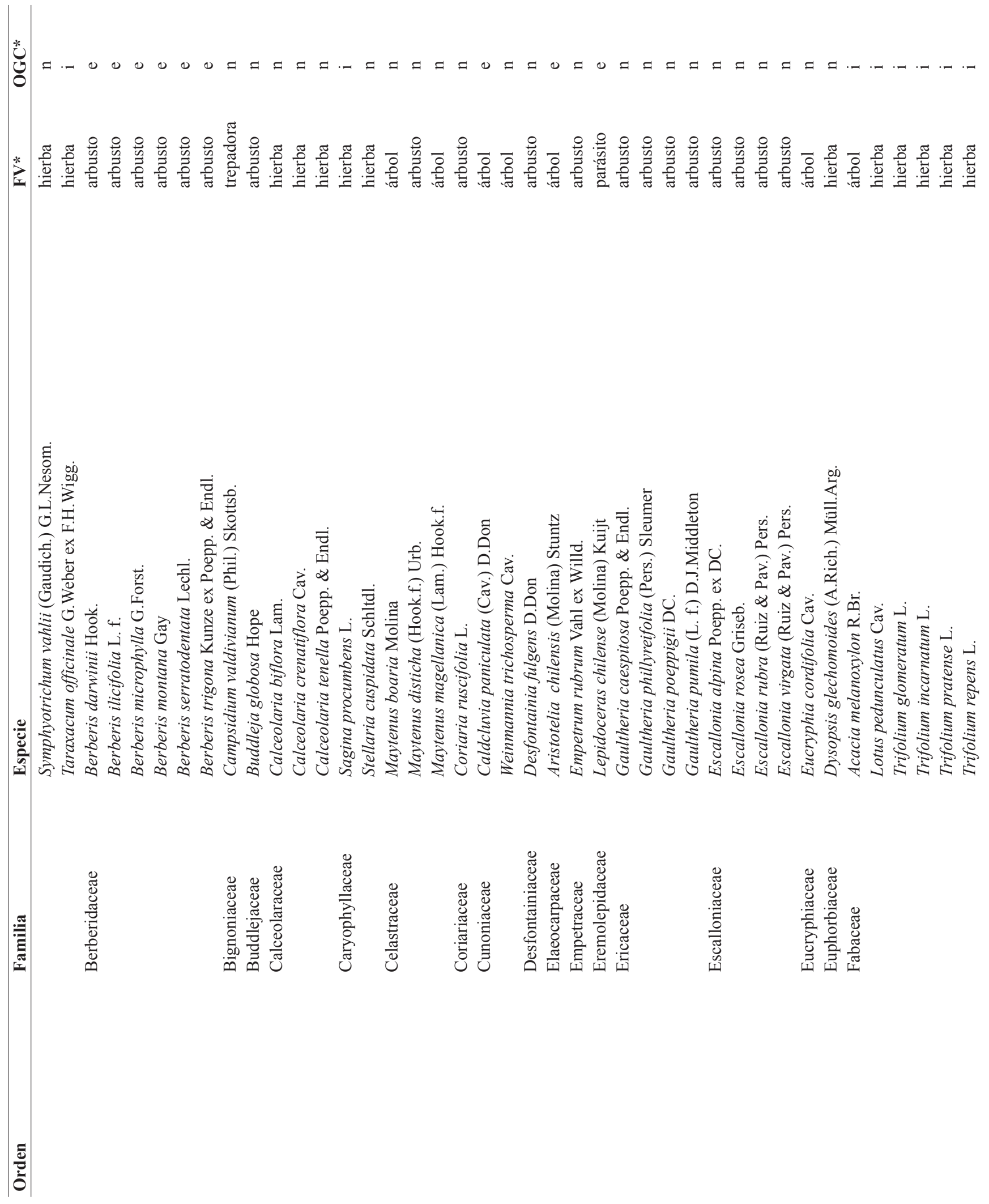




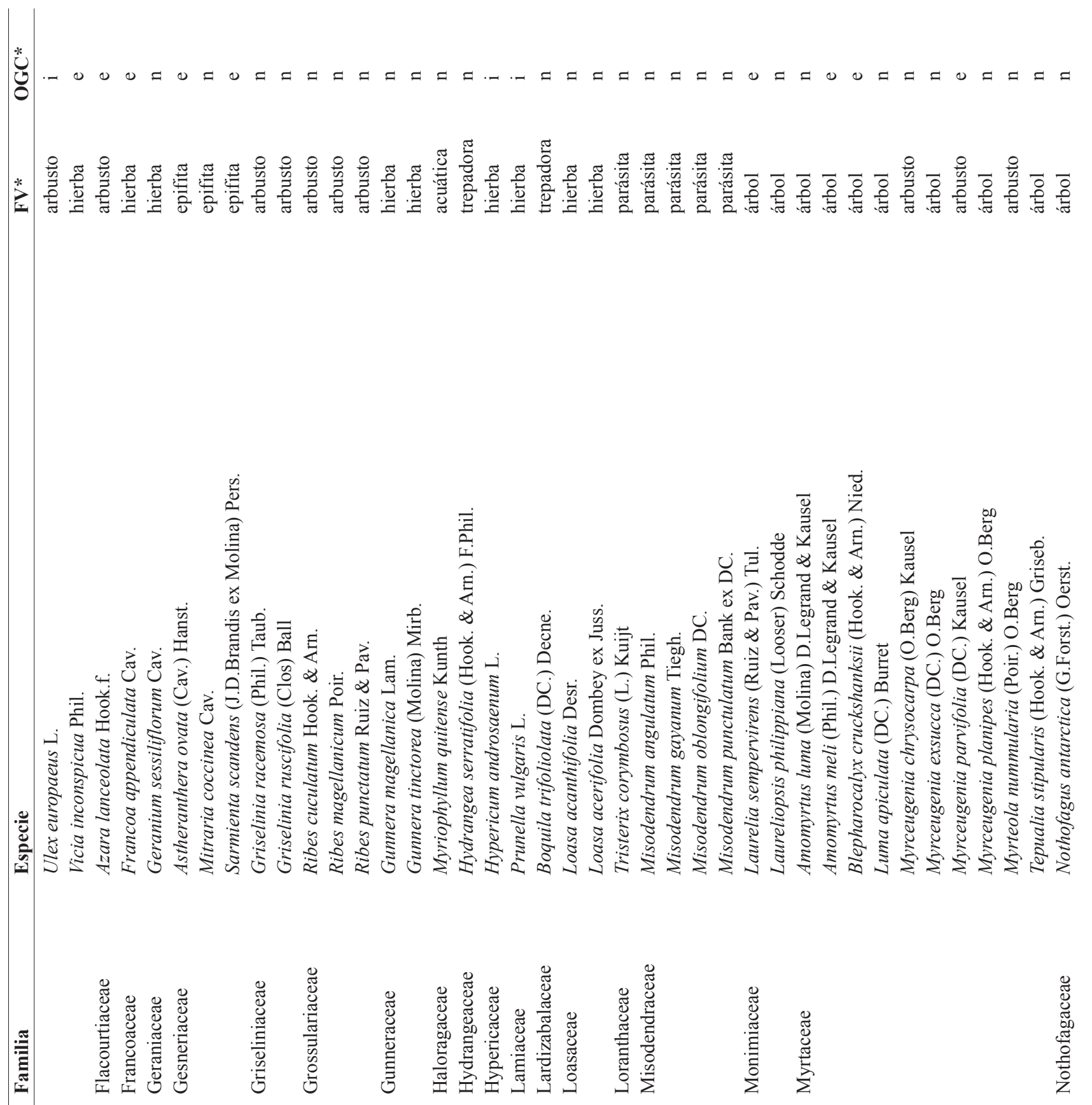




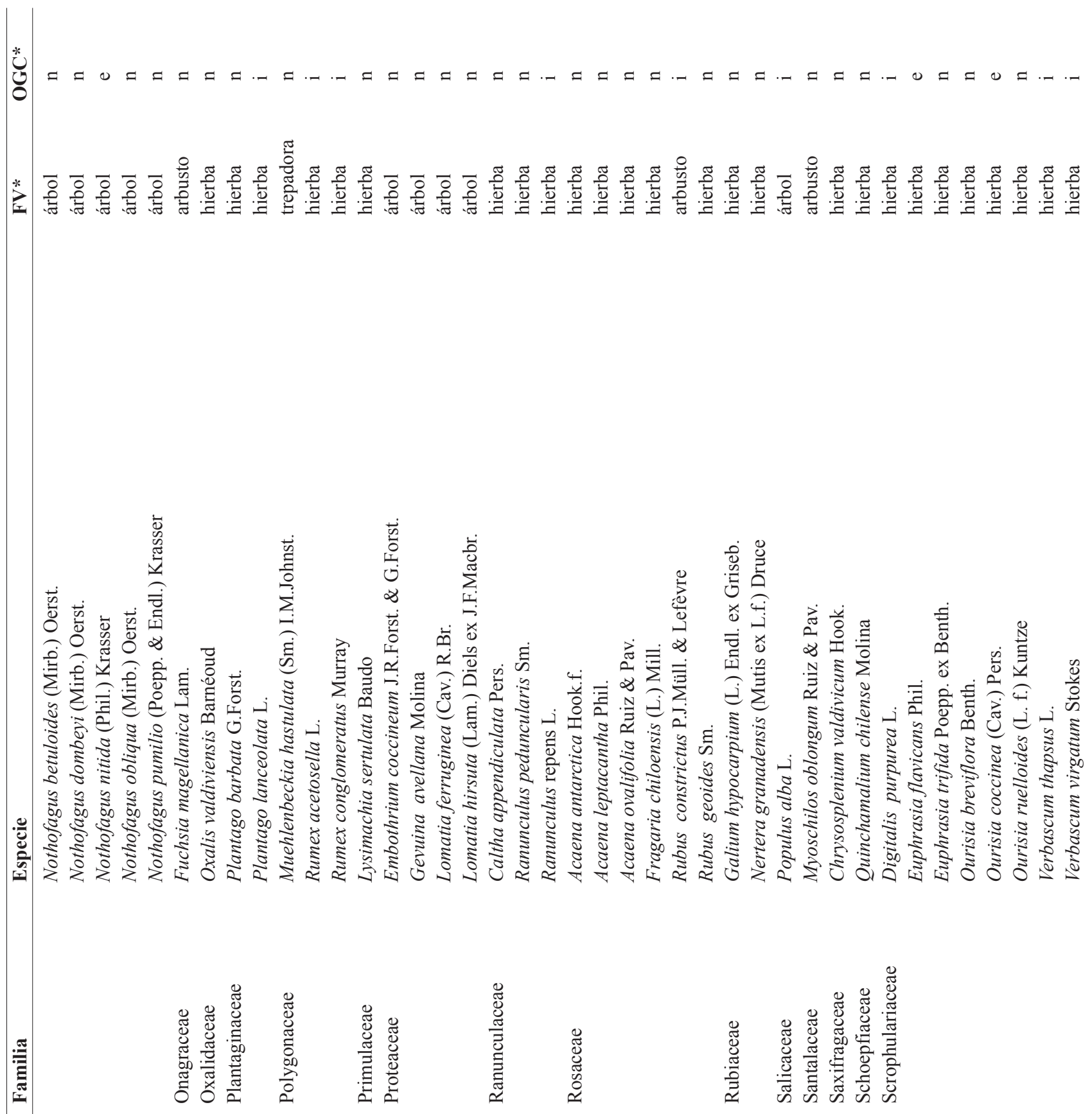




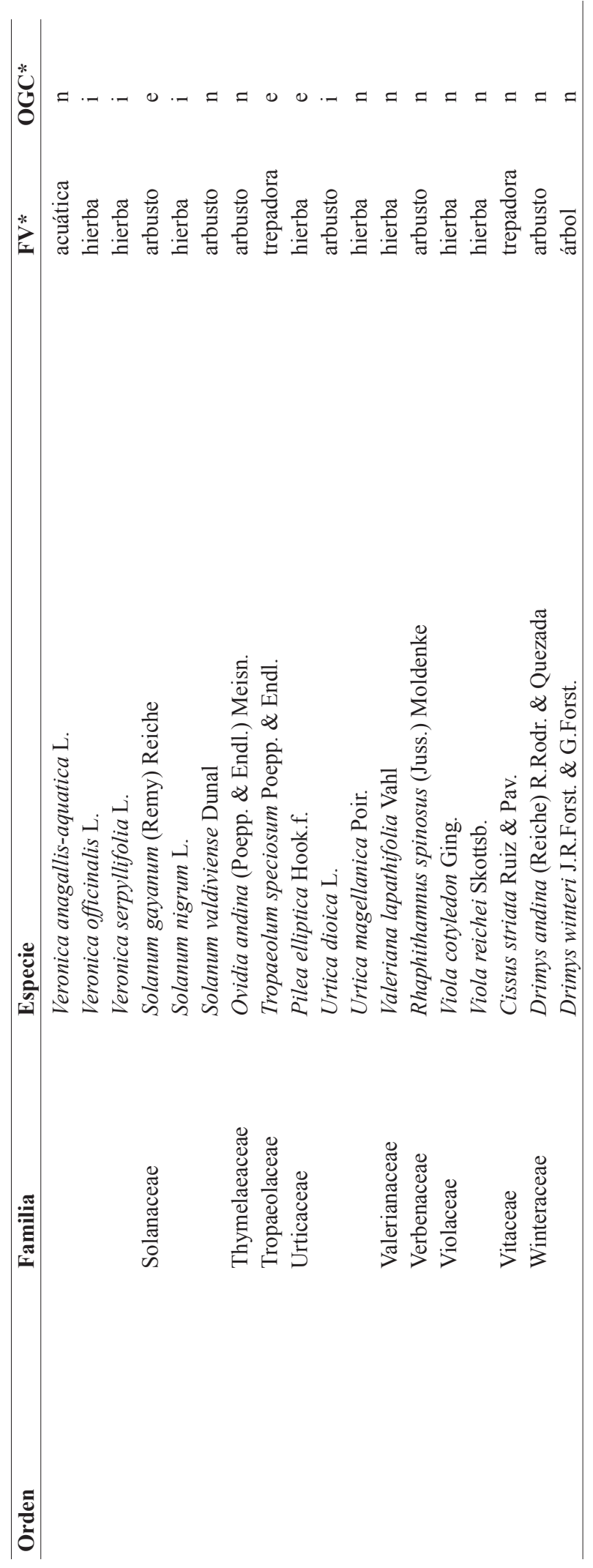




\section{AGRADECIMIENTOS}

Al Parque Futangue y Andrés Martínez (administrador), por el financiamiento y apoyo logístico; Ignacio Díaz por la elaboración del mapa (Fig 1); E. Hauenstein, por facilitar sus trabajos; a los alumnos en práctica y a los guardaparques por su valiosa colaboración en las campañas de terreno; a los revisores anónimos por sus apreciables aportes.

\section{BIBLIOGRAFÍA}

Armesto, J.J., C. Villagrán \& M.T.K Arroyo. 1996. Ecología de los bosques nativos de Chile. Editorial Universitaria, Santiago Chile. 470 pp.

Arroyo, M.T.K., L. Cavieres, A. Peñalosa, M. Riveros \& A. FAGGi. 1996. Relaciones fitogeográficas y patrones regionales de riqueza de especies en la flora del bosque lluvioso templado de Sudamérica.71-99 pp. Cap 4 en: Armesto, J.J.; C. Villagrán y M.T.K Arroyo (Eds) Ecología de los bosques nativos de Chile. Editorial Universitaria, Santiago Chile. 470 pp.

Baeza, M., E. Barrera, J. Flores, C. Ramírez \& R. Rodríguez. 1998. Categorías de conservación de Pteridophyta nativas de Chile. Boletín del Museo Nacional de Historia Natural 47: 23-46.

Benoit, I.L. (ed), 1989. Libro rojo de la flora terrestre de Chile. CONAF Santiago de Chile. 157p.

BECERRA, P. \& L. FaÚNDEZ. 1999. Diversidad florística de la Reserva Nacional Malalcahuello, IX Región, Chile. Chloris Chilensis 2(1) INTERNET: http://www.chlorischile.cl

Becerra, P. \& G. Cruz. 2000. Diversidad vegetacional de la Reserva Nacional Malalcahuello, IX Región de Chile. Bosque 21(2): 47-68.

Castro, S., J. Figueroa, M. Muñoz-Schick \& F. Jacksic. 2005. Minimum residence time, biogeographical origin, and life cycle as determinants of the geographical extent of naturalized plants in continental Chile. Diversity and Distribution 11: 183-191.

Domínguez, E., C. Marticorena, A. Elvebakk \& A. Pauchard. 2004. Catalogo de la flora vascular del Parque Nacional Pali Aike, XII Región, Chile. Gayana Botanica 61(2): 2772.

Donoso, C. 1981. Tipos Forestales de los Bosques Nativos de Chile. Documento de Trabajo $\mathrm{N}^{\circ}$. 38. Investigación y Desarrollo Forestal (CONAF, PNUD-FAO) (Publicación FAO Chile). 70 pp.

Fuentes-Ramírez A., A. Pauchard \& E. Hauenstein. 2011. Composición de la flora de praderas andinas en la Reserva Nacional Alto Bío-Bío (Lonquimay-Chile) y su relación con el régimen de pastoreo. Gayana Botanica 68: 28-39.

Gajardo, R. 1994. La Vegetación Natural de Chile. Clasificación y Distribución Geográfica. Editorial Universitaria, Santiago de Chile. 165 pp.

GonzÁlez, A. 2000. Evaluación del recurso vegetacional en la cuenca del río Budi, situación actual y propuestas de manejo. Tesis Licenciatura en Recursos Naturales. Escuela de Ciencias Ambientales, Facultad de Ciencias, Universidad Católica de Temuco. 87 pp.
Hauenstein, E., C. Ramírez, M. Latsague \& D. Contreras. 1988. Origen Fitogeográfico y espectro Biológico como medida del grado de intervención antrópica en comunidades vegetales. Medio Ambiente 9(1): 140-142.

Hechenleitner, P., M. Gardner, P. Thomas, C. Echeverría, B. Escobar, P. Brownless \& C. Martínez. 2005. Plantas Amenazadas del Centro-Sur de Chile. Distribución, Conservación y Propagación. Primera edición. Universidad Austral de Chile y Real Jardín Botánico de Edimburgo Valdivia $188 \mathrm{pp}$.

Luebert, F. \& P. Becerra. 1998. Representatividad vegetacional del Sistema Nacional de Áreas Silvestres Protegidas del Estado (SNASPE) en Chile. Ciencia y Ambiente 14(2): 62-69.

Luebert, F. \& P. Pliscoff. 2006. Sinopsis bioclimática y vegetacional de Chile. Editorial Universitaria, Santiago de Chile. 316 pp.

Lyndenmayer, D. \& J. Franklin. 2002. Conserving Forest Biodiversity. A comprehensive multiscaled approach. Island Press Washington, USA. 351pp.

Marticorena, C. 1991. Contribución a la estadística de la flora vascular de Chile. Gayana Botánica 47: 85-113.

Martínez, O. 1985. Aspectos de la flora y vegetación del Parque Nacional "Vicente Pérez Rosales" (Llanquihue - Chile). Bosque 6(2): 83-92.

Matthei, O. 1995. Manual de las malezas que crecen en Chile, Alfabeta Impresores. Santiago de Chile. 547 pp.

Moreno, R. 2011. Flora del Parque Futangue, Lago Ranco, Región de los Ríos. Tesis de pregrado, Ing. Forestal, Fac. Cs. Forestales y Recursos Naturales, UACh. Valdivia, Chile. $65 \mathrm{pp}$.

Muñoz, M. 1980. Flora del Parque Nacional Puyehue. Editorial Universitaria S.A. Santiago de Chile. 557p.

Myers, E., R. Mittermeier, C. Mittermeier, G. Da Fonseca \& J. Kent. 2000. Biodiversity hotspot for conservation priorities. Nature 403: 853-858.

Oberdorfer, E. 1960. Pflanzensoziologische Studien in Chile. Ein Vergleich mit Europa. Flora et vegetatio mundi 2: 1-208

Oltremari, J. \& K. Thelen. 2003.Planificación de áreas silvestres protegidas. Un manual para la planificación de áreas protegidas en Chile con especial referencia a áreas protegidas privadas. CONAMA- FAO. Santiago de Chile. $169 \mathrm{pp}$.

Pauchard, A. \& P. Alaback. 2004. Influence of elevation, land use, and landscape context on patterns of alien plant invasions along roadsides in protected areas of south central Chile. Conservation Biology 18: 238-248.

Pisano, E. 1956. Esquema de clasificación de las comunidades vegetales de Chile. Agronomía 2: 30-33.

Possingham, H., K. Wilson, S. Andelman \& C. Vynne. 2006. Protected areas: goals, limitations, and design. In: M. Groom, G. Meffe \& C.R. Carroll (eds.), Principles of Conservation Biology. Cap. 14: 509-551. Third edition. Sinauer Associates, Inc. Sunderland, Massachusetts, U.S.A 793 pp.

Primack R., R. Rozzi, P. Feinsinger, R. Dirzo \& F. Massardo. 2001. Fundamentos de conservación biológica, perspectivas latinoamericanas. Fondo de Cultura Económica, México. $497 \mathrm{pp}$

Rodríguez, R., D. Alarcón \& J. Espejo. 2009. Helechos nativos 
del centro y sur de Chile. Guía de campo. Ed. Corporación Chilena de la Madera, Concepción, Chile. 212 pp.

Schmithüsen, J. 1956. Die raunmliche Ordnung der chilenischen Vegetation. Bonner Geographische Abhandlungen 17: 1-86.

Serey, I., M. Ricci \& C. Smith-Ramírez. 2007. Libro Rojo de la Región de O’Higgins. Corporación Nacional Forestal Universidad de Chile, Rancagua, Chile, 222 pp.

Simonetti, J., M.T.K. Arroyo, A. Spotorno \& E. Lozada. 1995. Diversidad biológica de Chile. CONICYT. 364 pp.

UICN. 2010. UICN Red list of threatened species. Version 2010.4. http://www.iucnredlist.org (Julio 2, 2010).

Squeo, F.A., G. Arancio \& J.R. Gutiérrez. 2008. Libro Rojo de la Flora Nativa y de los Sitios Prioritarios para su Conservación: Región de Atacama. Ediciones Universidad de La Serena, La Serena. 456p.

Squeo, F.A., G. Arancio \& J.R. Gutiérrez. 2001. Libro Rojo de la Flora Nativa y de los Sitios Prioritarios para su Conservación: Región de Coquimbo. Ediciones Universidad de La Serena, La Serena, Chile. 456 pp.

Squeo, F.A., L. Cavieres, G. Arancio, J. Novoa, O. Matthei, C. Marticorena, R. Rodríguez, M.T.K. Arroyo \& M.
MuÑoz. 1998. Biodiversidad de la flora vascular en la Región de Antofagasta, Chile. Revista Chilena de Historia Natural. Nat. 71: 571-591.

Smith-Ramírez, C., P. Pliscoff, S. Tellier \& E. Barrera. 2005. Patrones de riqueza y distribución de la flora vascular de la Cordillera de la Costa de Valdivia, Osorno y Llanquihue, Chile. Cap. 14 en: Smith-Ramírez C., J. Armesto y C. Valdovinos (Eds.) Historia, biodiversidad y Ecología de los bosques costeros de Chile. Ed. Universitaria, Santiago de Chile. 253-277 pp.

Ter Steege, H., M. Jansen-Jacobs \& V. Datadin. 2000. Can botanical collections assist in a National Protected Area Strategy in Guyana. Biodiversity and Conservation 9(2): 215-240

VAN ANDEL,T. 2001. Floristic composition and diversity of mixed primary and secondary forest in northwest Guyana. Biodiversity and Conservation 10: 1645-1682.

Zuloaga, F., O. Morrone \& M. Belgrano. 2009. Catálogo de las plantas vasculares del Cono Sur Versión base de datos en sitio web del Instituto Darwinion, Argentina. URL: http:// www.darwin.edu.ar/Proyectos/FloraArgentina/FA.asp .

Recibido: 17.11.11

Aceptado: 11.12.12 